\title{
CONSIDERACIONES SOBRE LA FUERZA DE LAS RAZONES EN CONTRA DE DAÑAR
}

\author{
Santiago Truccone Borgogno \\ University of Graz \\ santiago.truccone-borgogno@uni-graz.at
}

RESUMEN: En este trabajo realizaré afirmaciones sobre la fuerza de las razones en contra de dañar. Distinguiré diferentes tipos de estados de daño y de acciones dañosas. Explicaré qué tipo de estado de daño es más grave y qué tipo de acción dañosa genera razones más fuertes en contra de dañar. Finalmente compararé la fuerza de las razones en contra de dañar derivadas tanto de los estados de daño como de los distintos tipos de acciones dañosas, para establecer una regla de prioridad entre ellas.

PALABRAS CLAVE: estados de daño, acciones dañosas, problema de la no identidad, comparabilidad, imprecisión

SUMMARY: In this work, I present some considerations on the strength of the reasons against harming. I distinguish between different kinds of harmed states and harming actions, and explain which kind of harmed state is worse, and which kind of harming action provides stronger reasons against harming. Finally, I compare the strength of the reasons against harming provided by harmed states and those provided by the different kinds of harming actions in order to establish a priority rule between them.

KEY WORDS: harmed states, harming actions, non-identity-problem, comparability, imprecision

\section{Introducción}

Entre todas las razones existentes en contra de dañar, aquellas derivadas de los estados de daño y aquellas derivadas del tipo de acción que ha causado un daño tienen un lugar preponderante. Las razones derivadas de los estados de daño parecen sugerir que, cuanto peor sea el daño sufrido, más fuertes serán las razones en contra de dañar. Por su parte, las razones derivadas del tipo de acción dañosa parecen sugerir que, si una acción causa un daño que el sujeto afectado no hubiese sufrido en ausencia de dicha acción, las razones en contra de dañar serán más fuertes que aquellas que existen en contra de causar un daño cuando el sujeto afectado hubiese sufrido un daño idéntico de todos modos. 
Creo que, aunque las afirmaciones anteriores son bastante plausibles, requieren afirmaciones adicionales. Para obtener una guía clara sobre la fuerza de las razones en contra de dañar, hace falta un desarrollo sobre cómo identificar cuándo un daño es más grave que otro, y sobre cuándo un tipo de acción dañosa genera razones más fuertes en contra de realizarla que las que generan acciones de otro tipo. En este trabajo haré afirmaciones tanto sobre los estados de daño como sobre las acciones dañosas. En relación con los estados de daño sostendré, primero, que una concepción de daño que combine una noción contrafáctica y una noción de umbral de daño es inevitable. Segundo, en cuanto a la gravedad de los daños, sostendré que, aunque puede afirmarse que un tipo de daño es más grave que otro, la comparación entre ellos sólo puede ser imprecisa. En relación con las acciones dañosas distinguiré entre aquellas que causan que un sujeto sufra un daño que sólo puede haber sido causado por tal acción (no redundante) y aquellas que causan un daño que el sujeto afectado hubiese sufrido aun en ausencia de dicha acción (redundante). Afirmaré que el primer tipo de acción dañosa genera razones más fuertes en contra de dañar que las del segundo tipo. Finalmente, sostendré que las razones en contra de realizar acciones dañosas redundantes que causan daños muy graves son más fuertes que las razones en contra de realizar acciones dañosas no redundantes que causan daños muy leves. Si mi propuesta resulta exitosa, contaremos con criterios claros con los que graduar la fuerza de las razones en contra de dañar en diferentes clases de casos.

\section{Estados de daño}

\subsection{Concepto}

Cuando alguien se encuentra en un estado de daño, diremos que está sufriendo un daño. ${ }^{l}$ Para propósitos morales, los estados de daño relevantes son aquellos que son el producto de una acción dañosa. Ambos elementos, estados de daño y acciones dañosas, son los componentes de los diferentes enfoques sobre dañar. Existen dos enfoques enfrentados que intentan explicar qué significa dañar. Por un lado, los enfoques relativos a los efectos afirman que una persona daña a otra en virtud de los efectos de la acción dañosa sobre el bienestar de un individuo. Por otro lado, las nociones relativas a la

\footnotetext{
${ }^{1}$ No interesan aquí consideraciones sobre el dolor, la angustia o experiencias conscientes.
} 
acción afirman que una persona daña a otra en virtud de la diferencia que una acción marca en el bienestar de una persona (Gardner 2017, pp. 3-6). Con respecto a los estados de daño, estos dos enfoques se diferencian en lo siguiente. Para los enfoques relativos a los efectos, un sujeto sufre un daño en virtud del tipo de estado en el que se encuentra; mientras que los enfoques relativos a la acción sostienen que para afirmar que un sujeto sufre un daño es necesario que la acción que causó tal estado de cosas haya empeorado el estado en que se encuentra el sujeto afectado.

La noción más ampliamente aceptada sobre los estados de daño es aquella que entiende que un sujeto está sufriendo un daño cuando se encuentra en un estado peor que otro estado en el que esa persona habría estado si no se hubiese actuado con relación a esa persona del modo en que se hizo (Feinberg 1984, p. 31). Esta concepción sobre los estados de daño, comúnmente denominada contrafáctica, se asocia usualmente a las nociones sobre dañar relativas a la acción. Para afirmar que alguien sufre un daño, de acuerdo con tal noción, se requiere que una acción haya empeorado el estado en que se encuentra el sujeto afectado. Esta clase de daños tienen esencialmente una dimensión comparativa (Parfit 2017, p. 134). Aunque esta noción es útil para identificar por qué se está sufriendo un daño en un gran número de situaciones, es incapaz de explicar por qué alguien está sufriendo un daño en ciertos casos donde parece claro que el sujeto afectado se encuentra en un estado de daño.

La noción de daño contrafáctica asociada al enfoque sobre dañar relativo a la acción es incapaz de proporcionar una respuesta satisfactoria en aquellos casos que atraviesa el que se conoce como problema de la no identidad. Este problema surge porque, en determinados casos, parece que la identidad de los afectados por ciertas acciones es irrelevante a la hora de calificar nuestras acciones como dañosas (Parfit 1987, cap. 16). El asunto radica en el hecho referido a que dependiendo de cuál sea la acción que se realice, la identidad de la persona resultante será diferente. Como esto es así, entonces, dichas acciones no pueden empeorar el estado en el que estarán los sujetos que existirán como consecuencia de ellas. Como, en estos casos, las acciones no pueden empeorar el estado en el que estarán los afectados, los enfoques sobre dañar relativos a la acción no pueden explicar por qué los sujetos afectados están sufriendo alguna clase de daño. Veamos el siguiente ejemplo:

Caso I (María): Marta acaba de regresar de Centroamérica. Acude al médico porque pretende concebir un hijo. El médico 
le informa que se ha contagiado del virus del Zika y que, si concibe ahora, su hijo nacerá con microcefalia. El médico le aconseja que espere un mes antes de concebir. De ese modo se asegurará que el virus desaparezca y su hijo no nazca con esa condición. Sin embargo, Marta hace caso omiso al consejo del médico y concibe a su hijo antes de transcurrido el mes. Como resultado, nace María con microcefalia y cierto retraso en sus capacidades intelectuales.

¿Está María sufriendo un daño? Dado que María nace con cierto retraso en sus capacidades intelectuales, parece que está sufriendo un daño. Sin embargo, no puede sostenerse que la acción que causó que María se encuentre en tal estado haya empeorado algún estado o condición en la que ella habría estado de otro modo. Si Marta hubiese esperado un mes para concebir, María no estaría en un mejor estado; en ese caso, nunca habría existido. Esto es así porque cada persona surge de un espermatozoide y de un óvulo particular. Por lo tanto, si una persona no hubiese sido concebida en el espacio de un mes alrededor del momento en que fue concebida, de hecho, nunca habría existido (Parfit 1987, pp. 351-355). ${ }^{2}$ Es decir, si María no hubiese sido concebida en el espacio de un mes con respecto al momento en que fue concebida, de hecho, nunca habría existido. Podría haber existido en su lugar otra persona que hubiese sido el hijo de Marta, pero esta criatura no habría sido María.

Dado que la acción de Marta no ha empeorado ningún estado en el que se encuentra María, para explicar por qué esta última está sufriendo un daño tenemos que abandonar los enfoques sobre dañar relativos a la acción. Debemos adoptar, en cambio, un enfoque relativo a los efectos. De acuerdo con este último tipo de enfoques, primero deben identificarse aquellos estados que merecen ser considerados como estados de daño, para luego afirmar que cualquier acción que los haya causado es una acción dañosa. Una aproximación que puede ayudarnos a identificar los estados de daño en casos como el analizado es la noción de umbral de daño (Meyer 2016). Obviamente, esto requiere que sea posible especificar un nivel de bienestar respecto del cual se vulnera el derecho de una persona si no cumplimos con

${ }^{2}$ El asunto no radica en negar la afirmación de que, por ejemplo, si yo hubiese sido concebido en otro momento, entonces, sería menor la probabilidad de que contrajera cierta enfermedad. En cambio, el problema es que si no hubiese sido concebido en el momento en que fui concebido (o al menos dentro del mes en que fui concebido), yo no existiría. En ese caso, mis padres podrían haber tenido un hijo, pero ya que ese hijo no se formó a partir del par de células del que me forme yo, ese hijo no sería yo. 
nuestro deber negativo de no causar que esa persona esté por debajo de dicho estándar (Meyer y Roser 2009, p. 229). Un modo plausible de especificar tal nivel es apelando a la noción de necesidades básicas. Así, el umbral estaría fijado levemente por encima del nivel en el que las personas tienen sus necesidades básicas satisfechas. ${ }^{3}$ Esta clase de daños son intrínsecos en el sentido de que su maldad no requiere que las personas estén en un estado peor que en el alternativo (Parfit 2017, p. 133).

La noción de umbral de daño nos permite explicar por qué María se encuentra en un estado de daño. Al nacer con cierto retraso intelectual, ella se encuentra por debajo del umbral y, por lo tanto, está sufriendo un daño. La acción de Marta, dado que ha causado que María se encuentre por debajo del umbral, es una acción dañosa, independientemente de que dicha acción no haya empeorado el estado en el que se encontraría María. Esta última condición no es necesaria para los enfoques sobre dañar relativos a los efectos. Aunque la noción de umbral de daño (referida a los estados de daño) como parte de un enfoque sobre dañar relativo a los efectos permite explicar este caso de modo satisfactorio, no permite dar cuenta de otros casos en los que creemos que se ha dañado a alguien. Veamos la siguiente situación:

Caso II (Pedro): Petra se somete a un proceso de diagnóstico prenatal mediante el cual trata de controlar el estado de salud de la criatura que porta en su seno. El médico le informa que los estudios han mostrado que su hijo es genéticamente perfecto y, por lo tanto, si el embarazo continúa en sus carriles normales, su hijo tendrá no sólo un riesgo ínfimo de padecer cáncer, problemas cardíacos o Alzheimer, sino también una inteligencia superior.

Petra tiene planeado realizar un viaje de placer al sur de Argentina. El médico le aconseja que no realice tal viaje dado que el feto se encuentra en una etapa importante de su desarrollo. Si no reposa durante unas semanas, la consecuencia será que el hijo que porta en su seno - Pedro - pierda cierto grado de inteligencia superior.

\footnotetext{
${ }^{3}$ Podemos entender por "necesidades básicas" aquellas que las personas tienen por el mero hecho de ser humanos; por ejemplo, alimentación, supervivencia, salud, etc. Debido a que las necesidades son básicas, tienen una gran importancia moral (Griffin 1986, p. 42).
} 
Petra piensa que no vale la pena sacrificar su viaje por el bienestar de su niño, de modo que hace caso omiso del consejo del médico y viaja al sur de Argentina. Efectivamente, el estrés del vuelo ocasiona que Pedro nazca con una inteligencia mayor de la que se considera normal, pero menor a la inteligencia superior que habría tenido.

¿Está Pedro sufriendo un daño? No podemos afirmar que Pedro está sufriendo un daño si apelamos a la noción de umbral de daño. Nacer con inteligencia mayor de la que se considera normal no puede estar por debajo de ningún umbral de daño definido de modo plausible. ${ }^{4}$ Sin embargo, parece que sí está sufriendo un daño. Después de todo, Pedro se encuentra en un estado peor que otro en el que habría estado si su madre hubiese obrado de modo diferente. Parece que para explicar por qué Pedro está sufriendo un daño tenemos que abandonar no sólo la noción de umbral de daño sino también el enfoque relativo a los efectos sobre dañar. Pero si esto es así, no podremos explicar por qué María (caso I) está sufriendo un daño y por qué su madre la ha dañado.

Aunque es verdadero que la noción de umbral de daño no puede explicar por qué Pedro está sufriendo un daño, no es necesario abandonarla. Ella puede ser combinada con una noción contrafáctica sobre los estados de daño como parte de un enfoque sobre dañar relativo a los efectos. Es decir, aunque es cierto que el umbral de daño no permite explicar por qué Pedro está sufriendo un daño, no es necesario afirmar que él ha sido dañado porque una acción ha empeorado el estado en el que se encuentra. Todo lo que es necesario afirmar es que Pedro se encuentra sufriendo un daño porque se encuentra en un estado tal que, si dicho estado no hubiese tenido lugar, él estaría en uno mejor. Esta última afirmación parece sospechosamente similar a la inicial contrafáctica relativa a la acción. Sin embargo, esto no es así. Olvidemos por un momento la noción de umbral de daño y quedémonos con ambas versiones contrafácticas:

[A] Noción contrafáctica de los estados de daño relativa a la acción:

${ }^{4}$ Es necesaria una aclaración aquí. Sigo a Eduardo Rivera López en su defensa del uso del concepto "salud normal". Él afirma que tal concepto se asume preteóricamente en muchos contextos, como cuando los médicos lo usan en relación con la salud de un recién nacido. Al mismo tiempo, él afirma que este concepto es contextual, en el sentido de que lo que es normal ahora puede no serlo en el futuro o en otro lugar (Rivera-López 2009, p. 347). 
Una persona $P$ sufre un daño sólo si está en un estado $E$ tal que si el agente no hubiese actuado con respecto a $P$ como lo hizo, $P$ estaría en un estado mejor.

[B] Noción contrafáctica de los estados de daño relativa a los efectos:

Una persona $P$ sufre un daño sólo si está en un estado $E$ tal que, si $\mathrm{E}$ no se hubiese producido, $P$ se habría encontrado en un estado mejor.

El siguiente caso puede ayudarnos a identificar la diferencia entre las dos nociones:

(Daños previos) Supóngase que un criminal quiere robar la tienda de joyas de Joaquín. Dado que el robo será más fácil si Joaquín no está en ella, el criminal contrata a un matón para que le quiebre las piernas el día anterior al robo. Cuando el matón llega a la casa de Joaquín, sin embargo, encuentra que el usurero local ya le ha roto las piernas. ${ }^{5}$

En esta clase de casos de acuerdo con la noción contrafáctica de los estados de daño relativa a la acción (y a cualquier noción de los estados de daño relativa a la acción), no es posible afirmar que Joaquín está sufriendo un daño. Joaquín no está en ningún estado tal que, si el usurero no hubiese actuado con respecto a él como lo hizo, él estaría en un mejor estado. Por lo tanto, conforme a esta noción, Joaquín no está sufriendo un daño. Si Joaquín no está sufriendo un daño, no puede considerarse que la acción del usurero lo ha dañado, por más que el primero tenga las piernas rotas a consecuencia de la acción del segundo. Esto no parece plausible. Claramente Joaquín, al tener las piernas rotas, está sufriendo un daño.

No obstante, si apelamos a la noción contrafáctica de los estados de daño relativa a los efectos, no existen problemas para afirmar que Joaquín está sufriendo un daño. Joaquín se encuentra en un estado tal que, si dicho estado se no hubiese producido, él habría estado en uno mejor. Es decir, Joaquín se encuentra en un estado en el que tiene las piernas rotas, y si el estado en el que tiene las piernas rotas no se hubiese producido, él estaría mejor. De esta manera podemos sostener que la acción del usurero local que ha causado que Joaquín sufra un daño es dañosa. Del mismo modo, esta aproximación puede

${ }^{5}$ He modificado el ejemplo de Hanser (2008, p. 434). 
explicar por qué Pedro (caso II) está sufriendo un daño. Al tener una inteligencia normal, Pedro se encuentra en un estado tal que, si dicho estado no se hubiese producido, él habría estado en uno mejor, es decir, Pedro habría tenido una inteligencia superior. De esta manera podemos explicar por qué Pedro sufre un daño sin necesidad de abandonar un enfoque sobre dañar relativo a los efectos. Sin embargo, como el enfoque contrafáctico no nos permite afirmar —ni siquiera en la versión relativa a los efectos- que María (caso I) está sufriendo un daño, tenemos que mantener también la noción de umbral de daño para obtener resultados satisfactorios en ambos casos. Creo que debemos adoptar, por lo tanto, una noción combinada de los estados de daño relativa a los efectos ${ }^{6}$ según la cual:

Combinada: una persona sufre un daño sólo si:

[a] está en un estado por debajo del umbral, o

[b] está en un estado $E$ tal que si $E$ no hubiese tenido lugar, esa persona se habría encontrado en un estado mejor.

\subsection{Comparabilidad de los daños}

La noción combinada de daño relativa a los efectos permite identificar los diferentes estados en los que creemos que un sujeto sufre un daño. Dicha noción nos proporciona dos condiciones que nos permiten afirmar que, si alguna de ellas ha sido satisfecha, el sujeto afectado

\footnotetext{
${ }^{6}$ Para otras razones en favor de las nociones combinadas, véase Meyer 2016; para nociones relativas a los efectos, véase Gardner 2017. En la construcción que aquí se propone, tanto para la noción de umbral como para la noción de contrafáctico, se asume la llamada tesis de la lista objetiva de bienestar. Esta tesis supone que hay determinados bienes cuya posesión incrementan el bienestar y cuya carencia lo hacen disminuir, independientemente de componentes subjetivos del agente tales como la satisfacción de preferencias o el placer. Para una descripción y discusión de los defectos y bondades de estas posiciones véase Parfit 1987 (pp. 493-502). No creo, empero, que la adopción de alguna de las posiciones subjetivistas alternativas sea incompatible con la noción de daño aquí defendida. Por ejemplo, si se adoptara como tesis de bienestar una posición que descansara en la satisfacción de preferencias, podría afirmarse que una persona sufre un daño basado en el umbral cuando se encuentra en un estado en el que cierto nivel de preferencia no es satisfecho y que, en cambio, sufre un daño basado en el contrafáctico cuando, de otro modo, sus preferencias se habrían satisfecho en mayor grado. Consideraciones similares pueden aplicarse a las nociones que descansan en la idea de felicidad. No obstante, debo reconocer que la noción combinada, junto con alguna tesis subjetiva de bienestar, haría que la comparación entre daños fuera mucho más compleja. Agradezco a un árbitro anónimo por insistir en este punto.
} 
estará sufriendo un daño. Sin embargo, ¿es más grave sufrir un daño por encontrarse por debajo del umbral de daño (condición [a]), o es más grave sufrir un daño por encontrarse en un estado tal que si dicho estado no hubiese tenido lugar, el sujeto afectado habría estado en uno mejor (condición [b])? Y si un sujeto se encuentra en un estado donde se satisfacen ambas condiciones, ¿es por ese hecho el daño sufrido más grave?

Antes de responder a estas preguntas debe notarse que existen dos modos en que los estados de daño pueden diferir en gravedad: cuantitativamente y cualitativamente. Dos estados de daño difieren cualitativamente cuando uno satisface la condición [a] de la noción combinada y el otro satisface la condición [b]. Cuando alguien esté sufriendo un daño que satisface la condición [a], afirmaremos que el tipo de daño que está sufriendo está fundado en el umbral (en adelante FU), dado que el estatus de daño deriva del hecho de que el nivel de bienestar de la persona afectada es menor que aquel que tiene derecho a tener. Cuando alguien esté en un estado de daño que satisface la condición [b], diremos que el tipo de daño que esta persona está sufriendo está fundado en el contrafáctico (en adelante FC), dado que el estatus de daño deriva de una comparación entre dos estados posibles: el estado en el que está la persona y aquel estado en el que la persona habría estado si el estado en el que se encuentra no se hubiese producido.

Para graduar la gravedad de los daños es de utilidad introducir la noción de unidades de daño. Cada unidad de daño debe ser medida en relación con una línea de base definida. De este modo tenemos, por un lado, unidades de daño fundadas en el umbral (FU) y, por otro lado, unidades de daño fundadas en el contrafáctico (FC). En relación con los daños $\mathrm{FU}$, mientras mayor sea la distancia entre el estado en que está la persona y el umbral de daño, mayor será el número de unidades de daño FU sufridas. Del mismo modo, en cuanto a los daños FC, mientras mayor sea la distancia entre el estado en que la persona afectada se encuentra y el estado contrafáctico en el que habría estado si el estado en que está no se hubiese producido, mayor será el número de unidades de daño $\mathrm{FC}$ sufridas.

Es importante tener presente que, del mismo modo que hay dos sentidos diferentes de daño, hay dos sentidos diferentes de unidades de daño. Las unidades de daño FC se usan para medir la gravedad de los daños FC, mientras que las unidades de daño FU se usan para medir la gravedad de los daños FU. Esto es importante porque, como discutiremos luego, las unidades de daño FU no tienen el mismo valor que las unidades de daño FC. 
Existen algunas circunstancias donde es relativamente sencillo poder identificar qué daño es más grave. Primero, no parece haber problemas en sostener que si dos sujetos - sin diferencias relevantes entre ellos- sufren daños cualitativamente idénticos, pero uno de ellos sufre un daño cuantitativamente mayor, esta persona estará sufriendo un daño más grave que el otro. Segundo, si dos personas sufren daños cuantitativamente idénticos, pero uno de ellos sufre un daño FU (condición [a]) y el otro uno FC (condición [b]), el primero estará sufriendo un daño peor que el segundo. Esto se puede explicar porque, sea donde sea que se haya marcado el nivel del umbral de daño, el sujeto que está sufriendo daños FU tendrá un nivel de bienestar mucho menor que aquel que está sufriendo daños FC. Esto parece una razón en favor de la afirmación de que sufrir un daño FU es peor que sufrir un daño $\mathrm{FC}$. Si, en los casos que hemos estado analizando, María (caso I) y Pedro (caso II) sufren daños cuantitativamente idénticos, pero María tiene un coeficiente intelectual muy bajo y Pedro uno normal, parece que María está sufriendo un daño peor, por más que Pedro (y no María) haya podido estar en un mejor estado. ${ }^{7}$ Finalmente, si dos personas se encuentran sufriendo un daño FU idéntico, pero una de ellas sufre además cierta cantidad de unidades de daño FC, parece que esta última está sufriendo un daño peor. Esto se puede defender pues, aunque ambos sujetos sufren un mal por encontrarse debajo del umbral de daño, uno de ellos sufre - además - el mal de tener un bienestar que es menor que aquel que habría tenido si el estado en el que se encuentra no hubiese tenido lugar. Imaginemos que nace un sujeto - Juan - en el mismo estado por debajo del umbral que María. Sin embargo, a diferencia de María, Juan podría haber nacido en un estado que se ubica en el umbral de daño. En este caso, claramente Juan está sufriendo un daño peor que María. Él puede tener dos reclamos, en tanto que María sólo tiene uno (véase Harman 2009, p. 146).

A la par de las circunstancias anteriores en que es relativamente sencillo identificar qué tipo de daño es más grave, existen otras en que el asunto incrementa su dificultad. Imaginemos que, respecto de las capacidades intelectuales, hemos fijado en 90 puntos de coeficiente intelectual (CI) el umbral de daño. ${ }^{8}$ Así, cualquiera que esté en un

\footnotetext{
${ }^{7}$ Para defender tal afirmación podemos apelar a una concepción como la del "suficientismo" que afirma que la preocupación por las personas que están debajo del umbral tiene prioridad sobre la preocupación por cualquier persona que esté por encima del umbral (Crisp 2003, p. 762).

${ }^{8}$ Parece razonable fijar el umbral de daño en 90 puntos de CI, dado que una
} 
estado por debajo de esa marca estará sufriendo un daño FU. Ahora imaginemos los casos siguientes:

\begin{tabular}{|c|c|c|}
\hline & Estado real & Estado posible \\
\hline Juan & 70 & 92 \\
\hline María & 49 & - \\
\hline Umbral de daño: 90 \\
\hline
\end{tabular}

En este caso, Juan y María están sufriendo un daño cuantitativamente diferente. Mientras que María sufre 41 unidades de daño FU, Juan sufre 20 unidades de daño FU y 22 de daño FC. ${ }^{9}$ Hemos dicho que sufrir cierta cantidad de unidades de daño FU es peor que sufrir la misma cantidad de unidades de daño FC. Imaginemos que le asignamos el valor 1 a cada unidad de daño FC y que cada unidad de daño FU vale el doble que cada unidad de daño FC. Como María sufre 41 unidades de daño FU, la gravedad del daño sufrido sería de $82(41 \times 2)$. Como Juan sufre 20 unidades de daño FU y 22 de FC, la gravedad del daño sufrido sería $62(20 \times 2+22)$. Por lo tanto, con base en estas consideraciones, María estaría sufriendo un daño peor que el que sufre Juan, lo que parece plausible.

Aunque esta posición parezca razonable, tiene ciertos inconvenientes. Primero, ¿̨cómo saber exactamente cuánto más grave es un tipo de daño en función de otro? En el ejemplo hemos supuesto que los daños FU son el doble de graves que los FC. Sin embargo, ¿por qué no afirmar que son sólo 1.5 más graves, o 0.6? Cualquier especificación en estos términos parece arbitraria. Segundo y más importante, esta clase de teorías es problemática cuando examinamos otra clase de situaciones. Consideremos a Pedro, quien nació con un CI de 90 y, de no ser por como actuó su madre, habría tenido un CI de 175:

puntuación entre 90 y 109 de coeficiente intelectual se considera de una inteligencia normal (Weiss 2006, p. 75).

${ }^{9}$ Estoy asumiendo que cada punto de CI es igual a una unidad de daño. Siendo el CI algo medible, parece un buen lugar para comenzar a someter a prueba la plausibilidad de la idea aquí desarrollada. Si ésta es exitosa, luego su análisis se puede extender a otros casos donde parezca más difícil realizar el tipo de comparaciones que haré aquí. 


\begin{tabular}{|l|c|c|}
\hline & Estado real & Estado posible \\
\hline Juan & 70 & 92 \\
\hline María & 49 & - \\
\hline Pedro & 90 & 175 \\
\hline Umbral de daño: 90 & \\
\hline
\end{tabular}

Supongamos que aceptamos que cada unidad de daño FU es el doble de grave que cada unidad de daño FC. Si esto es así, tenemos que afirmar que Pedro es quien está sufriendo el peor daño. El daño que sufre vale $85(85 \times 1)$, mientras que el daño que sufre María es de $82(41 \times 2)$. Esto no puede ser posible. No es posible que tener un CI de 90 (aun cuando se hubiera tenido uno mayor) sea peor que tener un CI de sólo 49 puntos. Préstese atención a que no podemos resolver completamente el problema si les asignamos un valor mayor a los daños FU que a los daños FC. Si le asignamos una prioridad muy alta a la gravedad de los daños $\mathrm{FU}$ en relación con la de los $\mathrm{FC}$, debemos confrontar el problema inverso. La versión más extrema de esta idea implica asignarles una prioridad lexical a los daños FU en relación con los FC. Esta posición afirma que si bien es siempre peor sufrir más unidades de daño FC, ninguna cantidad de unidades de daño FC puede ser tan grave como sufrir una sola unidad de daño FU. Bajo este esquema, cuanto más lejos del umbral de daño estén los sujetos, más graves serán los daños sufridos. Apelar a esta idea nos permite afirmar que María - 4l unidades de daño FUsufre un daño peor que Juan — daño FU de 20 unidades - quien sufre, a su vez, un daño peor que Pedro - ninguna unidad de daño $\mathrm{FU}$ - Sin embargo, el problema que nos genera es igualmente grave. Consideremos a María 2 , quien nació con un CI de 89, de manera tal que sufre 1 unidad de daño FU:

\begin{tabular}{|l|c|c|}
\hline & Estado real & Estado posible \\
\hline Pedro & 90 & 175 \\
\hline María $_{2}$ & 89 & - \\
\hline Umbral de daño: 90 & \\
\hline
\end{tabular}

En este caso, si consideramos que los daños FU son lexicalmente más graves que los FC, tenemos que afirmar que María 2 está sufriendo un daño (no sólo peor, sino) lexicalmente peor que el que sufre 
Pedro. Esto tampoco puede ser verdadero. Si la diferencia entre el nivel de bienestar de Pedro y María 2 es de sólo 1 punto y Pedro sufre - adicionalmente - 85 unidades de daño FC, María, quien está sufriendo un daño FU de sólo 1 unidad, no puede estar sufriendo un daño (lexicalmente) peor que el que sufre Pedro. En estas circunstancias, parece que el daño sufrido por María $a_{2}$ no es peor que el que sufre Pedro. Esta tesis nos lleva a afirmar que si una persona tiene un nivel de bienestar ligeramente menor que otra, pero esta segunda persona - a diferencia de la primera - habría tenido un nivel de bienestar mucho mayor si el estado en el que se encuentra no se hubiese producido, la primera persona está sufriendo un daño (lexicalmente) peor que la segunda.

Así, parece que, a la hora de especificar cuánto más grave es un tipo de daño con respecto al otro, nos encontramos con un dilema. $\mathrm{Si}$ asignamos una prioridad menor a la gravedad de los daños $\mathrm{FU}$ que a la de los FC, tenemos que afirmar que Pedro (daño FC de 85) sufre un daño peor que María (daño FU de 41). Piénsese, por ejemplo, que si en vez de referirnos a diferentes tipos de CI, nos referimos a recursos económicos, podríamos vernos obligados a afirmar que un multimillonario al que se le ha sustraído una gran parte de su capital (esto es, que de otro modo sería mucho más que multimillonario) sufre un daño peor que quien no tiene recursos suficientes para satisfacer sus necesidades básicas. Esto es inaceptable. En cambio, si le asignamos una prioridad muy grande o una prioridad lexical a la gravedad de los daños FU por encima de la de los FC, tenemos que afirmar que María 2 (CI de 89 y sólo 1 unidad de daño FU) sufre un daño (lexicalmente) peor que Pedro (CI de 90 y 85 unidades de daño FC). Esto tampoco parece aceptable.

\subsection{Imprecisión en la comparabilidad}

Creo que el problema surge porque hemos comparado la diferencia en gravedad de los daños de modo incorrecto. Para graduar la gravedad de los daños en los casos en los que los sujetos afectados sufren daños tanto cuantitativa como cualitativamente diferentes, tenemos que apelar a lo que se conoce como "concepción lexical imprecisa" (Parfit 2016, p. 112). En nuestro contexto, exige afirmar que sufrir el tipo de daño fundado en el umbral es lexicalmente peor que sufrir el tipo de daño fundado en el contrafáctico, pero que es peor — sólo - de modo impreciso. La tesis es la siguiente: aunque sufrir más unidades del tipo de daño fundado en el contrafáctico hace que el daño sufrido sea más grave, sufrir un número suficiente de 
unidades del tipo de daño fundado en el umbral hará que el daño sufrido sea siempre peor que sufrir cualquier número de unidades del tipo de daño fundado en el contrafáctico. Para defender esta tesis, resulta necesario, en primer lugar, explicar qué significa que una comparación sea imprecisa y, en segundo, por qué ése es el tipo de comparación que debe utilizarse entre los daños FU y FC.

Una comparación es precisa cuando puede afirmarse que uno de dos elementos es mejor, peor o igual que otro haciendo referencia a una escala de unidades en función de la cual los elementos son clasificados (Chang 2016, p. 185). Este tipo de comparaciones suele ejemplificarse bajo el rótulo de modelo lineal, dado que se comparan dos ítems o estados como posiciones en una línea (Parfit 2016, p. 114). Éste es el modelo que utilizamos cuando realizamos una comparación cuantitativa entre daños de la misma clase. En estos casos es posible afirmar no sólo que sufrir un número mayor de unidades de daño es peor que sufrir un número menor, sino también cuánto más grave es. Uno modo de saber si una comparación responde al modelo lineal es el siguiente: recordemos el caso de Pedro e imaginemos a alguien que padece una situación similar a la de Pedro, pero con un daño cuantitativamente menor. Llamemos a este sujeto Pedroz. Como Pedro sufre 85 unidades de daño FC, mientras que Pedro, por ejemplo, 40, Pedro sufre un daño peor que Pedro, . Ahora imaginemos un continuo que va de Pedro a Pedro 2 , tal que cada Pedro subsiguiente sufre una unidad menos de daño FC. Si comparamos el daño que sufre Pedro 2 con el de otros sujetos que sufren daños FC ligeramente menores que Pedro tal que Pedro ${ }^{1}$ sufre 84 unidades, Pedro $^{2}$ 83, y así sucesivamente, tendremos que encontrar un Pedro ${ }^{N}$ en dicho continuo que sufra un daño igual al que sufre Pedroz. Una implicación práctica importante de este tipo de comparaciones es que genera una relación transitiva entre dos ítems. Esto es, si Pedro 2 sufre un daño igual de grave que Pedro $^{N}$ y Pedro sufre un daño peor que Pedro ${ }^{N}$, entonces Pedro sufre un daño peor que Pedroz.

Ahora bien, cuando comparamos daños FU con daños FC no parece que pueda ser aplicable este modelo lineal. ¿Cuántas unidades del tipo de daño fundado en el contrafáctico son necesarias para afirmar que el daño sufrido es igual a sufrir $X$ cantidad de unidades del tipo de daño fundado en el umbral? No parece existir una escala con la que podamos medir la diferencia de gravedad entre daños cualitativamente distintos. En estos casos podemos apelar, en cambio, a una comparación imprecisa. Una comparación es de este tipo cuando, aunque puede afirmarse que uno de dos elementos es mejor, 
peor o igual que otro en relación con un valor, no existe una escala de unidades en función de la cual se puedan clasificar los elementos (Chang 2016, p. 188). La idea es que no hay una fórmula numérica o tasa que nos permita afirmar cuánto más grave o peor es un elemento en comparación con otro.

Para comprender de mejor modo este tipo de comparaciones puede ser útil introducir un ejemplo parfitiano. Parfit sugiere que una de dos experiencias dolorosas puede ser el doble de mala que la otra si entraña sufrir un dolor de la misma intensidad por el doble de tiempo que la primera. En este caso, es posible hacer dicha afirmación dado que el valor relativo de cada experiencia dolorosa varía en función de una sola propiedad medible: la duración. Aquí, la duración es el estándar en relación con el cual puede medirse la gravedad de dichas experiencias. Si la intensidad del dolor es idéntica, entonces, una de dos experiencias será más grave que otra en función de cuán duradera sea. Si es el doble de duradera, será el doble de grave; sin embargo, imaginemos que las dos experiencias dolorosas difieren tanto en su duración como en su intensidad. En este último caso parece no haber una verdad precisa sobre si uno u otro sufrimiento es peor, y cuánto peor. No parece haber una escala de valor en función de la cual se pueda medir la importancia relativa de la intensidad y la duración (Parfit 2016, p. 113). Aquí, como las experiencias dolorosas difieren tanto en su duración como en su intensidad, tenemos que medir su gravedad en función de estas dos propiedades: intensidad y duración. Cuando tomamos dos experiencias dolorosas, una de las cuales es cada vez peor en intensidad, mientras que la otra lo es en duración, cada unidad de sufrimiento añadida cualitativamente diferente no sólo añadirá gravedad a la experiencia, sino también imprecisión en la comparación. Por lo tanto, aunque podamos afirmar que una experiencia dolorosa es peor o igual que la otra, no podrá serlo de manera precisa. Una consecuencia práctica -importante para el tema que nos ocupa- es que, a diferencia de lo que ocurre con las comparaciones precisas, cuando dos ítems son igualmente buenos (o malos) de modo impreciso, la relación que se genera entre ellos no es transitiva. Así, si $A$ es igualmente malo de modo impreciso que $B$ y $B$ es peor que $C$, no se sigue que $A$ sea peor que $C$. Si, en el ejemplo anterior, sufrir un dolor $A$ (de $X_{+1 \text { intensidad }}$ e $Y_{+20 \text { duración }}$ ) es imprecisamente igual de malo que sufrir un dolor $B\left(\right.$ de $X_{+10 \text { intensidad }}$ e $Y_{+4 \text { duración }}$ ) y sufrir un dolor $B$ es peor que sufrir un dolor $C$ (de $X_{+10 \text { intensidad }}$ e $\left.Y_{+3 \text { duración }}\right)$, no se sigue que sufrir un dolor $A$ sea peor que sufrir un dolor $C$. 
Pensemos nuevamente en los estados de daño. Sufrir un daño, hemos afirmado, es malo porque implica que el sujeto afectado se encuentra por debajo de una línea de base o estándar de bienestar relevante. Así, por ejemplo, uno de dos daños (cualitativamente idénticos) puede ser el doble de grave que el otro si el primero involucra sufrir $X$ número mayor de unidades de daño que el segundo. En estos casos, al igual que en la comparación entre dos experiencias dolorosas de la misma intensidad, la comparación sobre la gravedad de los daños depende de una sola línea de base: de cuán lejos estén los sujetos afectados del estándar relevante de bienestar. En estas situaciones, como hemos visto al comparar los daños sufridos por Pedro y Pedro 2 , es aplicable el modelo lineal; sin embargo, cuando dos estados de daño se diferencian cualitativamente, existen dos líneas de base en función de las cuales se mide la gravedad de los estados de daño. Tal como ocurre con las experiencias dolorosas en que tenemos que medir su gravedad tanto en función de cuán duraderas sean como en función de cuán intensas sean, aquí tenemos que medir la gravedad de los daños también en función de dos estándares: umbral de daño y vida contrafáctica que el sujeto afectado habría tenido si el estado en el que se encuentra no hubiese tenido lugar. Esto hace inaplicable el modelo lineal. Por lo tanto, cuando queremos comparar dos estados de daño que difieren tanto cualitativamente como cuantitativamente, cada unidad de daño cualitativamente diferente añadida agrega no sólo gravedad al daño sufrido, sino también imprecisión a la comparación.

Luego de estas aclaraciones, creo que ya estamos en condiciones de defender la concepción lexical imprecisa aplicada a los estados de daño. De acuerdo con la concepción lexical, sufrir el tipo de daños fundados en el umbral (FU) es lexicalmente más grave que sufrir el tipo de daño fundado en el contrafáctico (FC). Si la diferencia de gravedad entre daños FU y FC fuese precisa, dicha concepción implicaría que si bien es siempre peor sufrir más unidades de daño FC, ninguna cantidad de unidades de daño FC puede ser tan grave como sufrir una sola unidad de daño FU. Hemos visto que esta concepción es implausible dado que nos lleva a afirmar que María, quien sufre un daño FU de 1 unidad, está sufriendo un daño peor que Pedro, quien sufre 85 unidades de daño FC y cuyo bienestar es sólo 1 punto mejor que el de María 2 ; pero, como hemos argumentado, la comparación entre estados de daño FU y estados de daño FC es imprecisa. Por lo tanto, no estamos obligados a aceptar tal conclusión. Para evitarla tenemos que apelar a la concepción lexical imprecisa, según la cual, aunque sufrir más unidades de daño FC hace que el 
daño sufrido sea más grave, un número suficiente de unidades de daño FU siempre hará que el daño sufrido sea peor que cualquier número de unidades de daño FC.

¿Cómo evita esta concepción afirmar que María 2 sufre un daño peor que Pedro, y al mismo tiempo permite afirmar que María sufre un daño peor que Pedro? Sencillamente afirmando que el número de unidades de daño FU que sufre María 2 no es suficiente para sostener que ella está sufriendo un daño lexicalmente peor que Pedro, en tanto que el número de unidades de daño FU sufridas por María son suficientes para establecer tal prioridad lexical. Los casos son los siguientes:

\begin{tabular}{|c|c|c|}
\hline & Estado real & Estado posible \\
\hline María & 49 & - \\
\hline María 2 & 89 & - \\
\hline Pedro & 90 & 175 \\
\hline
\end{tabular}

Comencemos con la afirmación — que parece aceptable - de que María 2 no sufre un daño lexicalmente peor que Pedro: la cantidad de bienestar que tiene Pedro, en comparación con la que habría tenido si el estado en el que se encuentra no hubiese tenido lugar, parece impedir afirmar que el daño que sufre María 2 es lexicalmente peor que el que sufre Pedro. Recordemos que María ${ }_{2}$ sufre 1 unidad de daño FU y Pedro, además de sufrir 85 unidades de daño FC, se encuentra en un estado sólo 1 punto por arriba de aquel en el que está María $_{2}$. Ahora bien, imaginemos un continuo que va desde María, tal que cada María ${ }_{2}$ sucesiva sufre 1 unidad de daño FU más que María 2 : María $_{2-\text { sucesiva }}{ }^{l}$ sufre 2 unidades de daño FU, María ${ }_{2-\text { sucesiva }}{ }^{2}$ sufre 3 y así de manera consecutiva. En algún punto de este continuo tiene que haber una María ${ }_{2-s u c e s i v a}{ }^{N}$ que esté sufriendo un daño imprecisamente igual de grave que el que sufre Pedro, esto es, el estado en el que se encuentra María ${ }_{2-\text { sucesiva }}{ }^{N}$ tiene que representar un daño (imprecisamente) tan grave como el que representa para Pedro tener un bienestar de 85 unidades menor que aquel que habría tenido. Si seguimos agregando unidades de daño FU a María $2-$ sucesiva $^{N}$, tal que lleguemos, por ejemplo, a María (41 unidades de daño FU), será posible afirmar que el daño que ella sufre es peor que el que sufre Pedro (y que cualquier Pedro ${ }_{2}^{N}$ ). 
Esto no quiere decir que, para afirmar que sufrir un daño FU es lexicalmente peor que sufrir uno FC, estemos afirmando que una cantidad precisa $(2,3,10,40, \ldots)$ de unidades de daño FU sea lexicalmente peor que cualquier cantidad de unidades de daño FC. Si ése fuera el caso, entonces las comparaciones seguirían siendo precisas. Lo que afirmamos, en cambio, es que existe una zona en la que los diferentes estados de daño son igualmente graves de modo impreciso, aunque por fuera de dicha zona, los daños FU son lexicalmente peores que los daños FC. Por lo tanto, cuando llegamos a un número suficiente de unidades de daño FU, no importa cuántas unidades de daño FC se sufran, el daño FU siempre será peor. Esto es verosímil, pues sufrir un gran número de unidades de daño FU implica que la calidad de vida del sujeto afectado es tan baja que o bien no vale la pena ser vivida, o bien está cerca de ese nivel. ${ }^{10}$ De esta manera, sin abandonar una diferenciación lexical en cuanto a la gravedad de los daños FU con respecto a los FC, podemos explicar tanto por qué María (41 unidades de daño FU) sufre un daño peor que Pedro (85 unidades de daño FC), como por qué María 2 (1 unidad de daño FU) no sufre un daño peor que Pedro.

Una segunda consecuencia de la concepción lexical imprecisa es que la relación que se genera entre dos estados de daño cuando son imprecisamente igual de graves no es transitiva, de manera tal que en nuestro ejemplo anterior- aunque María ${ }_{2}$ sucesiva ${ }^{N}$ esté sufriendo un daño imprecisamente igual de grave que Pedro, y Pedro $^{N}$ esté sufriendo un daño más grave que Pedro(porque sufre un número $X$ de unidades de daño FC más que Pedro), no se sigue que Pedro ${ }^{N}$ esté sufriendo un daño más grave que María ${ }_{2-\text { sucesiva }}{ }^{N}$. Es posible que los daños que están sufriendo sigan siendo imprecisamente igual de graves.

¿Y cómo comparar casos donde un sujeto sufre un daño FU, y otro sujeto un daño FU y también FC, cuando no se encuentran en

\footnotetext{
${ }^{10}$ Una vida no vale la pena ser vivida cuando habría sido preferible no haber existido nunca a vivir esa vida. Por ejemplo, una vida de puro sufrimiento tiene que ser peor que no haber existido nunca. Esto no quiere decir ni que (1) no haber existido nunca habría sido mejor ni que (2) sería mejor que no se viviera ninguna vida que tenga sufrimiento. Afirmar (1) es irrazonable pues, en ese caso, no habría habido nadie que "no hubiese existido". Sin embargo, tener una vida de sufrimiento es algo que le pasa a un sujeto real y, por lo tanto, puede afirmarse que es malo (o muy malo) para él (véase Meyer 1997). Afirmar (2) también es problemático dado que toda vida contiene alguna dosis de sufrimiento, lo que haría que fuera mejor que ninguna vida fuera vivida. Aunque, por ejemplo, David Benatar afirma que en toda vida la dosis de sufrimiento es mayor que el beneficio que conlleva, por lo cual sería mejor que ninguna nueva vida fuera vivida (véase Benatar 2006, pp. 28-59).
} 
el mismo estado? Creo que existe un método relativamente sencillo para resolver este tipo de casos. Primero, nótese que en estos casos la imprecisión en la comparabilidad será generalmente menor que cuando comparamos estados de daño solo $\mathrm{FU}$ con estados de daño solo FC. A diferencia de ese caso, aquí los sujetos se encuentran en estados de daño que comparten la propiedad de estar por debajo del umbral de daño. Por lo tanto, la diferencia de gravedad entre los daños sufridos sólo puede radicar en la diferencia de gravedad en las unidades de daño cualitativamente diferentes adicionales. Así, para saber quién sufre un daño que sea peor, primero, tenemos que restar las unidades de daño cualitativamente idénticas y luego comparar - de acuerdo con el procedimiento anterior - la gravedad relativa de las unidades de daño cualitativamente disímiles. Así, cuando las unidades de daño FU resultantes (luego de la resta de unidades de daño cualitativamente idénticas) sean suficientes, se podrá afirmar que el daño que sufre uno de los afectados es lexicalmente peor al que sufre el otro, sin importar cuántas unidades de daño FC esté sufriendo el segundo. Hasta ese punto, también encontraremos una zona donde los daños podrán ser imprecisamente igual de graves, o donde la cantidad de unidades de daño FC sufridas impliquen que el sujeto sufre un daño más grave que el que sufre un sujeto que se encuentra en un estado donde no sufre una cantidad suficiente de unidades de daño FU.

\section{Acciones dañosas}

Hasta aquí hemos realizado consideraciones sobre los estados de daño. Aún nos resta analizar el segundo elemento de las nociones sobre dañar: las acciones dañosas. Existen dos enfoques opuestos sobre las acciones dañosas. Por un lado, las tesis no comparativas afirman que, para que una acción sea considerada dañosa, es suficiente que cause un daño a alguien. Este tipo de acciones dañosas integran lo que hemos llamado enfoque sobre dañar relativo a los efectos. Por otro lado, las tesis comparativas sobre la acción dañosa afirman que para que una acción se considere dañosa debe empeorar el estado en el que se encuentra el ser afectado. Este tipo de acciones dañosas integran lo que hemos denominado enfoque sobre dañar relativo a la acción.

En el apartado anterior hemos afirmado que es preferible sostener un enfoque sobre dañar relativo a los efectos que un enfoque relativo a la acción. Esto nos permite identificar de mejor manera aquellos casos donde creemos que alguien está sufriendo un daño. Sin embargo, dado que supone una noción no comparativa sobre la acción dañosa, 
tal enfoque debe enfrentar algunas objeciones. Autores como Derek Parfit han afirmado que, para que una acción sea considerada dañosa - en el sentido de que existen razones en contra de realizarla-, tiene que ser verdadero que, si hubiésemos actuado de modo diferente, esa persona habría estado en una mejor condición (Parfit 1987, p. 69). Si esto no sucede, de acuerdo con su posición, el daño causado no aportará ninguna razón moral en contra de ocasionarlo. Aunque la posición de Parfit parece demasiado extrema dado que no parece ser algo moralmente irrelevante causar que alguien sufra un daño aun cuando esa persona no habría podido estar en una mejor condición, tal y como hemos afirmado al analizar los casos que el problema de la no identidad atraviesa, una versión más débil de su argumento puede ser más prometedora.

Fiona Woollard ha argumentado que, aunque existen razones en contra de realizar acciones dañosas no comparativas, las razones en contra de realizar tal tipo de acciones son más débiles que aquellas que existen en contra de realizar acciones dañosas comparativas $(2012$, p. 686). Su idea es que, si una acción dañosa no hace que el individuo afectado esté en una condición peor, beneficios importantes conferidos a otros pueden justificar la imposición de tales daños. En cambio, una igual cantidad de beneficios no pueden justificar la imposición de daños cuando la acción dañosa hace que la persona afectada esté en una condición peor (Woollard 2012, p. 687).

En este punto argumentaré — siguiendo a Molly Gardner (2017) — que Woollard falla en su tarea de mostrar que las acciones dañosas comparativas generan razones más fuertes en contra de dañar que las no comparativas. Para probar su argumento, Woollard se vale del siguiente ejemplo:

(Salvando a Sarah): Adam no le guarda ningún rencor en a Víctor. Barney está por disparar y matar a Víctor. Adam no tiene modo de prevenir esto. Sarah está por morir. Adam puede salvarla, pero hacerlo tendrá el efecto secundario de que matará a Víctor. Adam salva la vida de Sarah y mata a Víctor. (2012, p. 685; la traducción es mía.)

De acuerdo con Woollard, Adam tiene permitido salvar la vida de Sarah aun cuando la consecuencia de ello sea que Víctor muera. En este caso, la acción que causa que Víctor sufra un daño es de tipo no comparativa dado que dicha acción no empeora el estado en el que Víctor habría estado. En estos casos, según Woollard, las razones en contra de causar tal daño pueden ser superadas por la 
presencia de otras razones, por ejemplo, las derivadas del tamaño del beneficio que Sarah ha recibido (2012, p. 685). No obstante, si -en una segunda versión del caso - la muerte de Víctor lo colocase en un estado peor que otro en el que habría estado en ausencia de la acción dañosa, para Woollard, salvar a Sarah de un modo cuya consecuencia sea que Víctor muera no estaría permitido (2012, p. 686). Esto sería así porque, de no ser por la acción de Adam, Víctor habría estado en una mejor condición. Así, según Woollard, las acciones dañosas comparativas generan razones más fuertes en contra de dañar que las que generan las no comparativas. Es decir, aunque los dos tipos de acciones dañosas generen razones en contra de dañar, dado que las razones que aportan los beneficios obtenidos pueden superar sólo la fuerza de las razones en contra de realizar acciones dañosas no comparativas y no de acciones dañosas comparativas, entonces, las razones en contra de realizar acciones dañosas comparativas son más fuertes que las razones en contra de realizar acciones dañosas no comparativas.

Como ha puntualizado Molly Gardner (2017), el argumento de Woollard no muestra que las acciones dañosas comparativas generen razones más fuertes en contra de dañar que las acciones dañosas no comparativas. Que en la primera versión de Salvando a Sarah esté justificado que Adam salve a Sarah y no lo esté en la segunda versión no exige detenerse en consideraciones sobre acciones dañosas comparativas. No es necesario argumentar que está permitido salvar a Sarah en la primera versión, porque de ese modo no se causaría que Víctor esté en estado peor que otro en el que habría estado; en tanto que no lo está en la segunda versión porque esto no tiene lugar. Todo lo que se necesita afirmar es que, en la primera versión del caso, está permitido salvar a Sarah porque a Víctor se le causa un daño que habría sufrido incluso en ausencia de dicha acción. En cambio, no está permitido hacer esto en la segunda versión del caso porque el daño sufrido por Víctor sólo puede haber sido causado por la acción de Adam.

Molly Gardner llama redundante al tipo de acción presente en la primera versión del caso (Salvando a Sarah), y no redundante a la presente en la segunda versión $(2017$, p. 8). Definiremos las acciones dañosas redundantes como aquellas que causan que alguien sufra un daño que habría sufrido aun en ausencia de dicha acción. En tanto que las acciones dañosas no redundantes serán aquellas que causan un daño que sólo se puede haber sufrido a causa de tal acción. Por lo tanto, si es esta distinción (redundante/no redundante) y no la otra (comparativa/no comparativa) la que explica por qué está justificado 
salvar a Sarah cuando Víctor iba a morir aun en ausencia de dicha acción, y no lo está cuando Víctor no iba a morir en ausencia de tal acción, no se ha probado que las acciones dañosas comparativas generen razones más fuertes en contra de dañar que las no comparativas. Todo lo que se ha probado es que las acciones dañosas no redundantes generan razones más fuertes que las redundantes. Puede parecer que estas dos distinciones se traslapan. No obstante, esto no es así. Analicemos el siguiente caso:

Caso III - (Anna): Kate tiene una enfermedad terminal. Si no se somete a un tratamiento a base de células madre en los próximos años, morirá. El médico que la trata les comenta a sus padres que, afortunadamente, a través de la fertilización in vitro es posible concebir a un niño del que se pueden extraer tales células para salvar a Kate.

Steve selecciona un embrión para ser implantado sobre la base de una mutación que causará que el niño nazca con cierto retraso en sus capacidades intelectuales. Steve selecciona este embrión mediante una técnica que le permite extraerle ciertas células que harán que la vida de Kate se salve. Como resultado nace Anna con el mismo retraso en capacidades intelectuales que María (caso I).

Si Steve no hubiera seleccionado e implantado el embrión, Jeeves lo habría hecho y habría ocasionado que Anna naciera con el mismo retraso intelectual. Sin embargo, la técnica que habría empleado Jeeves no le permitiría extraer las células necesarias para salvar la vida de Kate. ${ }^{11}$

En esta primera versión del caso III, la acción de Steve, aunque causa un daño, no provoca que la víctima esté en un estado peor que otro en el que habría estado en ausencia de su acción. Por lo tanto, el tipo de acción realizada por Steve es no comparativa. Sin embargo - en una segunda versión del caso III-, si Jeeves no hubiese estado presente, la acción de Steve tampoco habría provocado que la víctima estuviera en un estado peor que otro en el que habría estado en ausencia de su acción. En esta segunda versión del caso, tal como ocurre con María (caso I), si Steve no hubiese actuado como lo hizo, el resultado no habría sido que Anna estaría en un estado mejor, sino sólo que no existiría. De manera tal que ambas acciones son de tipo no comparativo. En ninguna de las dos versiones del

${ }^{11}$ He modificado el ejemplo de Gardner 2017 (p. 10). 
caso III, la acción de Steve causa que Anna esté en un estado peor que otro en el que habría estado en ausencia de su acción; pero, aunque ambas acciones sean de este tipo, existe una diferencia entre ellas. Mientras que en la primera versión del caso la acción de Steve es redundante, en la segunda versión es no redundante. En la primera versión del caso III, Steve causa que Anna sufra un daño que habría sufrido aun en ausencia de su acción; en tanto que el daño que causa Steve en la segunda versión del caso III sólo puede haber sido producto de su acción. Por lo tanto, la distinción entre acciones dañosas comparativas y no comparativas es diferente de la distinción entre acciones dañosas redundantes y no redundantes.

Ahora bien, ¿pueden las razones derivadas del beneficio otorgado a Kate superar la fuerza de las razones en contra de que Steve cause el daño que sufrirá Anna? Podría pensarse que, tal como la acción de Adam está justificada en la primera versión de Salvando a Sarah y no lo está en la segunda, la acción de Steve está justificada en la primera versión del caso III, en tanto que no lo está en la segunda. Sin embargo, existe una diferencia entre los dos casos que hace difícil sostener tal analogía. Mientras que en Salvando a Sarah, la muerte de Víctor se produce como mera consecuencia de la acción de Adam de salvar a Sarah, en el caso III Steve quiere el daño de Anna como medio para salvar a Kate. Puede sostenerse entonces que en ninguna de las dos versiones del caso III (ni cuando la acción es no redundante ni cuando es redundante), la acción de Steve está justificada.

Cabría, sin embargo, hacerse otra pregunta. Imaginemos que, en lugar de ser alternativas, las dos versiones del caso III son sucesivas; es decir, primero tendrá lugar la versión donde la acción de Steve es redundante y luego la versión donde es no redundante. En cada uno de los casos, las víctimas serán dos personas diferentes: en el primero AnnaR (redundante) y en el segundo AnnaNR (no redundante). También imaginemos que podemos evitar una de las acciones de Steve. Podemos evitar la acción de Steve cuando es redundante o podemos evitarla cuando es no redundante. ¿Cuál decidiríamos evitar? Parece que, si decidimos evitar un tipo de acción, es porque hay razones más fuertes en contra de realizar ese tipo de acciones. Creo que deberíamos evitar la acción de Steve cuando es no redundante antes que cuando es redundante. Podemos sostener esto apelando al hecho de que, de ese modo, se evitará que alguien en el mundo sufra, hecho que no tiene lugar si evitamos la acción de Steve en la versión donde ésta es redundante. En otros términos, parece que tenemos que evitar la acción de Steve en la segunda versión del caso III cuando Jeeves no está presente debido a que menos personas sufren 
daños. Si estas afirmaciones son correctas, las razones en contra de realizar acciones dañosas no redundantes son más fuertes que las razones en contra de realizar acciones dañosas redundantes.

\section{Estados de daño y acciones dañosas}

Hemos comenzado este trabajo afirmando que las razones en contra de realizar acciones que causen que determinados sujetos experimenten daños son más fuertes en la medida en que los daños sufridos sean más graves. Al analizar los estados de daño hemos tratado de explicar cuándo puede considerarse que determinado daño es más grave que otro. Luego, hemos afirmado que las razones para no realizar acciones dañosas no redundantes son más fuertes que las existentes para no realizar acciones dañosas redundantes. De manera que, tomando en cuenta la gravedad de los estados de daño y los diferentes tipos de acciones dañosas, tenemos cuatro casos en los que la fuerza de las razones en contra de dañar difiere. Si tuviésemos que elaborar un ranking al respecto, el caso que ocupa el primer lugar y el que ocupa el cuarto son sencillos. El primer lugar es el del caso donde una acción dañosa no redundante ocasiona que alguien sufra un daño muy grave; el último, aquel donde una acción dañosa redundante ocasiona que alguien sufra un daño leve. Sin embargo, no está claro qué combinación entre estados de daño y acciones dañosas debe ocupar las posiciones segunda y tercera en la escala. Si se les da prioridad a las razones derivadas de los estados de daño, entonces, las razones en contra de realizar acciones dañosas redundantes que ocasionan daños graves son más fuertes que las derivadas de una acción dañosa no redundante que ocasiona un daño leve. En cambio, si se le da prioridad a la fuerza de las razones derivadas de los distintos tipos de acciones dañosas, el resultado es el inverso.

Ante el interrogante anterior, no puedo dar una respuesta concluyente aquí; no obstante, me gustaría sugerir algunas ideas para empezar a resolver este problema final. Para pensar en el asunto, sería de utilidad distinguir dos clases de casos: el primero, cuando la diferencia entre la gravedad de los daños causados por una u otra acción es muy grande; el segundo, cuando esto no ocurre. El primer caso sería aquel donde comparamos la fuerza de las razones en contra de dañar derivadas de acciones dañosas redundantes que causan daños muy graves con la fuerza de las razones en contra de dañar derivadas de acciones dañosas no redundantes que causan daños muy leves. El segundo caso sería aquel donde comparamos la fuerza de las razones en contra de realizar acciones dañosas redundantes que 
causan daños más graves que los que causa una acción dañosa no redundante, pero donde no existe una diferencia muy grande entre la gravedad de los daños causados por ambas acciones.

En relación con el primer caso, debe notarse que los daños sufridos a raíz de las acciones dañosas redundantes implican que los sujetos vean su nivel de bienestar gravemente afectado. Piénsese en algunas de las situaciones que consideramos más serias y graves en la historia de la humanidad. Por ejemplo, en las acciones realizadas por oficiales y personal militar durante la última dictadura militar en Argentina (1976-1983). Imaginemos que somos jueces y llega a nuestro juzgado un delito de esta naturaleza: nos llega el caso de un torturador-X cuyo crimen fue cometido durante la última dictadura militar argentina, y queda comprobado que si él no hubiese torturado, otro sujeto lo habría hecho. A la vez nos llega el caso de un sujeto-Y que ha lesionado a otro, pero la lesión sufrida por la víctima no podría haberse producido en ausencia de la acción del sujeto-Y. Ahora tenemos que imponerles una pena al torturador-X y al sujeto-Y. Aunque la acción dañosa del torturador-X sea redundante y la del sujeto- $Y$ sea no redundante, parece que la pena que debe recibir el torturador- $\mathrm{X}$ es mayor que la que debe recibir el sujeto-Y que lesiona. Si este razonamiento es plausible, parece que, al menos en los casos donde el daño causado es muy grave, las razones derivadas de los estados de daño deben tener prioridad $-\mathrm{y}$ de ese modo son más fuertescon respecto a las derivadas del tipo de acción dañosa. ${ }^{12}$

En el segundo tipo de situaciones el asunto es menos claro. Cuando la diferencia entre la gravedad de los daños causados por una u otra acción no es muy grande, no es evidente que las razones en contra de dañar derivadas de los estados de daño tengan prioridad sobre las razones derivadas de las acciones dañosas. En esta franja no parece que pueda sostenerse una prioridad absoluta de las razones en contra de dañar derivadas de los estados de daño, con respecto a las razones

${ }^{12}$ Esto no quiere decir que siempre que un daño sea muy grave, las razones en contra de realizar la acción que lo produjo serán más fuertes que las razones en contra de realizar cualquier acción alternativa. Para afirmar tal extremo deben tenerse en cuenta otras variables, como el costo - para el agente - de realizar cada acción, el número de acciones al alcance del agente, la probabilidad de que en cada acción se produzca cierto resultado, la intención y el conocimiento del agente, etc. El argumento se aplica sólo a aquellos casos donde comparamos acciones dañosas redundantes con no redundantes, todo lo demás es igual, y al realizar acciones del primer tipo se producen daños muy graves, y al realizar las del segundo tipo, muy leves; es decir, se asume que todas las otras variables reseñadas en esta nota se mantienen constantes. Debo agradecer a dos árbitros anónimos por ayudarme a explicar este punto. 
derivadas del tipo de acción dañosa presente. Pareciera que, al igual que al comparar estados de daño FU con estados de daño FC, aquí las razones derivadas de los estados de daño y aquellas derivadas de los distintos tipos de acción dañosa son imprecisamente comparables. Que esto sea así puede explicar por qué, cuando la diferencia entre los daños causados por uno u otro tipo de acción dañosa no es muy grande, nos encontramos en una zona donde no parece claro qué razones en contra de realizar uno u otro curso de acción tienen más fuerza. ${ }^{13}$

\section{BIBLIOGRAFÍA}

Benatar, D., 2006, Better Never to Have Been: The Harm of Coming into Existence, Oxford University Press, Oxford.

Chang, R., 2016, "Parity, Imprecise Comparability and the Repugnant Conclusion", Theoria, vol. 82, no. 2, pp. 182-214.

Crisp, R., 2003, "Equality, Priority and Compassion", Ethics, vol. 113, no. 4 , pp. $745-763$.

Feinberg, J., 1984, Harm to Others: The Moral Limits of Criminal Law, vol. I, Oxford University Press, Oxford.

Gardner, M., 2017, "On the Strength of the Reason Against Harming", Journal of Moral Philosophy, vol. 14, no. 1, pp. 73-87. DOI: 10.1163/ 17455243-46810043.

Griffin, J., 1986, Well-Being: Its Meaning, Measurement and Moral Impact, Oxford University Press/Clarendon Press, Oxford. DOI: 10.1093/ 0198248431.001 .0001$.

Hanser, M., 2008, "The Metaphysics of Harm", Philosophy and Phenomenological Research, vol. 87, no. 2, pp. 421-450.

Harman, L., 2009, "Harming as Causing Harm", en M. Roberts y D. Wasserman, Harming Future Peoples: Ethics, Genetics and the Non Identity Problem, Springer, Nueva York, pp. 137-151.

Meyer, L., 2016, "Intergenerational Justice", The Stanford Encyclopaedia of Philosophy (edición de verano de 2016), Edward N. Zalta (ed.), dis-

${ }^{13}$ Agradezco a Lukas H. Meyer, Harald Stelzer, Sabine Hohl, Pranay Sanklecha, Kian Mintz Woo, Daniel Petz y Seunghyun Song, de la Universidad de Graz, por ayudarme a mejorar los argumentos aquí expresados. A Axel Gosseries, Nenad Miscevic, Alessandro Pinzani, Eduardo Rivera López, Julio Montero, Gustavo Beade, Guillermo Lariguet, Fernando Lizárraga, Hugo Seleme, José Peralta, Juan Iosa, Paula Gastaldi y a dos árbitros anónimos por sus valiosos comentarios. También a Romina Frontalini Rekers, Ramiro Moyano, Lucas Misseri, Ignacio Gómez Perdiguero, Maite Zelaya, Cecilia Carrizo, Valeria Bizarro y María del Mar Castillo por reiteradas discusiones sobre el asunto. Por último, quisiera agradecer a CONICET (Argentina) y al Austrian Science Fund, sin cuyo apoyo económico no habría podido realizar este trabajo. 
ponible en: <http://plato.stanford.edu/archives/sum2016/entries/justiceintergenerational/ $>$ [consultado: 20/04/2017].

Meyer, L., 1997, "Can Actual Future People Have a Right to NonExistence?", Archives for Philosophy of Law and Social Philosophy, vol. supl. 67, Rights, pp. 200-209.

Meyer, L. y A. Gosseries (comps.), 2009, Intergenerational Justice, Oxford University Press, Oxford.

Meyer L. y D. Roser, 2009, "Enough for the Future", en Meyer y Gosseries 2009, pp. 219-248.

Parfit, D., 2017, "Future People, the Non-Identity Problem, and PersonAffecting Principles", Philosophy and Public Affairs, vol. 45, no. 2, pp. 118-157.

—_, 2016, "Can We Avoid the Repugnant Conclusion?", Theoria, vol. 82, no. 2, pp. 110-127.

- 1987, Reasons and Persons, Clarendon Press, Oxford.

Rivera-López, E., 2009, "Individual Procreative Responsibility and the Non-Identity Problem", Pacific Philosophical Quarterly, vol. 90, no. 3, pp. 336-363.

Woollard, F., 2012, "Have We Solved the Non-Identity Problem", Ethical Theory and Moral Practice, vol. 15, no. 5, pp. 677-690.

Recibido el 20 de abril de 2017; revisado el 2 de febrero de 2018; aceptado el 8 de febrero de 2018. 